\title{
Control of Vibration of Transmission Lines
}

\author{
Marcel Migdalovici, Tudor Sireteanu and Emil M. Videa \\ Institute of Solid Mechanics, Romanian Academy, Bucharest, Romania
}

\begin{abstract}
(Revised 22 October 2009; accepted 27 January 2010)
The problem of vibration control of overhead line conductors subjected to laminar transverse wind, which induces stationary vibrations by Kármán effect, is important due to the consequences on these structures lifetime and service. We consider the conductor (cable) model as the Euler-Bernoulli beam, fulfilling the authors' condition that detaches the conductor model of the beam model with viscous, hysteretic, or dry friction internal-damping hypothesis. The aeolian vibration control of the conductor is based on the energy-balance principle that takes accounts for the wind-energy input, the energy dissipated by the conductor due to hysteretic self-damping properties (or equivalent viscous damping) and, eventually, the energy dissipated by the Stockbridge dampers. The aim of this approach is to mitigate the vibration level of overhead line conductors. The original analytical expression of the free-vibration modes and the resonance-frequencies equation for the cable with clamped extremities have been produced. The analytical expression of the kinetic energy of the cable is compared with the amount of dissipated energy, obtained by experimental means, for the control of vibration of transmission lines. Some applications are presented here.
\end{abstract}

\section{INTRODUCTION}

We consider the cable model derived from the EulerBernoulli beam with viscous, hysteretic, or Coulomb internal damping. ${ }^{1-11}$ The analytical expression of the free-vibration modes and the resonance-frequencies equation for the cable with clamped extremities are produced using our hypothesis of the cable imposed to the Euler-Bernoulli beam, essentially for accurate identification of the cable-model parameters. Highlighted in this paper is the property of any Euler-Bernoulli beam model to be substituted with our cable model for sufficient high frequencies because our hypothesis of the cable is respected by the Euler-Bernoulli beam in these conditions. The classic analytical solutions of the Euler-Bernoulli beam equation are applicable for low frequencies, but for high frequencies (see the case of fuel bundle beams of the nuclear power plant), our cable model gives analytical solutions. We were able to find some recent studies in our domain of interest. ${ }^{12-21}$ Our experimental research was performed on a specialized stand endowed with an overhead conductor with clamped extremities, alone or with a choice of Stockbridge dampers, and mounted on the extreme zones of the span. The resonance frequencies and vibration modes of the conductor in the stand are also identified theoretically and experimentally. The possibility of analyzing the influence of the concentrated harmonic force, applied on the cable middle span, and the influence of the aeolian forces through their energy diagrams were discovered. This gives the possibility of using the energy-balance principle to determine the vibration level of the cable at the resonance and the dynamic bending strain of the cable, versus frequencies, in the domain of interest. The analytical aspects of the internal-damping terms influence versus frequencies in the cable models are discussed.

\section{MATHEMATICAL MODEL OF CABLE WITH GENERAL DAMPING}

The following equation of free vibrations is considered: ${ }^{1-7}$

$$
\begin{aligned}
m_{L} \frac{\partial^{2} w_{i}}{\partial t^{2}}= & -c_{i}^{H *} w_{i}-\left(c_{i}^{V}+\frac{c_{i}^{H}}{\omega_{i}^{V H}}\right) \frac{\partial w_{i}}{\partial t}+ \\
& +T \frac{\partial^{2} w_{i}}{\partial x^{2}}-E I \frac{\partial^{4} w_{i}}{\partial x^{4}}+q
\end{aligned}
$$

Equation (1) describes the behavior of the cable, excited by the force $q=q(x, t)$, applied transversal on the cable, acting in the point of abscissa $x$ at time $t$, on the viscous damping hypothesis by the constant coefficient $c_{i}^{V}$, on the hysteretic damping hypothesis by the constant coefficient of the form $c_{i}^{H} / \omega_{i}^{V H}$, and on the dry friction (Coulomb) damping hypothesis, expressed by the coefficient $c_{i}^{H *}$. The coefficient $c_{i}^{H *}$ is, piecewise, constant, as a function of time $t$, and the sign is such that the sign of the damping force $c_{i}^{H *} w_{i}(x, t)$ is opposite to that of the velocity $\dot{w}_{i}(x, t)=\partial w_{i}(x, t) / \partial t$ at any time $t$. Other explicit expression of the dry-friction force is $c_{i}^{H 1}\left|w_{i}(x, t)\right| \operatorname{sign}\left(\dot{w}_{i}(x, t)\right)$, where $c_{i}^{H 1}$ is constant. $^{7}$ The first expression of the dry-friction force is deduced, in our case, taking into account that the functions $w_{i}(x, t)$ and $\partial w_{i}(x, t) / \partial t$ continue with separable variables. We denote by $\omega_{i}^{V H}$ the circular frequency of order $i$ for damped free vibration, by $f_{i}^{V H}=\omega_{i}^{V H} / \pi / 2$ the resonance frequency of order $i$ for damped free vibration, by $m_{L}$ the mass unit length of the cable, by $E I$ the bending rigidity of the cable, by $T$ the tension in the cable, by $y_{i}(x, t)$ the corresponding vertical displacement of the cable for vibration mode of order $i$, and by $L$ the span length of the cable.

Firstly, we searched the stabilized free transverse vibrations of the cable without damping and with clamped extremities, which are of standing waves form:

$$
w_{r}(x, t)=w_{r}(x) \sin \left(\omega_{r} t+\varphi\right) .
$$

In Eq. (2), the notations signify that $\omega_{r}=2 \pi f_{r}$ is the circular frequency, with $f_{r}$ the resonance frequency of the cable in free vibrations without damping, and that $\varphi$ is the phase angle between the initial impulse and displacement. The following 